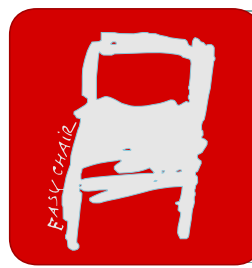

EPiC Series in Health Sciences

Volume 4, 2020, Pages 237-239

CAOS 2020. The 20th Annual Meeting of the International Society for Computer Assisted Orthopaedic Surgery

\title{
Automatic muscle elongation measurement during shoulder arthroplasty planning
}

\author{
J. Pitocchi ${ }^{1 *}$, K. Plessers ${ }^{1 *}$, M. Wesseling ${ }^{1}$, G.H. van Lenthe ${ }^{2}$, M. A. Pérez ${ }^{3}$ \\ ${ }^{1}$ Materialise NV, Leuven, Belgium \\ ${ }^{2}$ KU Leuven, Leuven, Belgium \\ ${ }^{3}$ University of Zaragoza, Spain \\ jonathan.pitocchi@materialise.be katrien.plessers@materialise.be
}

\section{Introduction}

Adequate deltoid and rotator cuff lengthening in total shoulder arthroplasty (TSA) is crucial to maximize the postoperative functional outcome and to avoid complications (Lädermann et al., 2014). Hence surgeons and patients could benefit from including muscle length information in preoperative planning software.

Although different methods have been introduced to automatically indicate patient-specific muscle attachment and wrapping points (Kaptein \& van der Helm, 2004; Marra et al., 2015), the definition of a fast and accurate workflow is still a challenge, due to the large variability in bone shapes.

Statistical shape modelling (SSM) has recently been used to automatically indicate landmark on target bones (Plessers et al., 2018). This method is less dependent on shape variability and could overcome the aforementioned limitation in accuracy. Therefore, the goal of this study is to develop and evaluate the accuracy of a novel automatic method for measuring deltoid and rotator cuff elongation during preoperative planning of shoulder arthroplasty, based on a statistical shape modelling approach.

\section{Materials and Methods}

A set of $40 \mathrm{CT}$-scans with full scapula and humerus was selected for the construction of the humerus SSM. Anatomical landmarks were manually indicated on the humerus models by two experts. Interoperator variability was assessed and the mean location of each landmark was considered as the ground-truth for the accuracy analysis. For the scapula, a previously developed SSM which had been validated for automatic landmarking, was used (Plessers et al., 2018).

First, humerus and scapula SSMs were used to automatically indicate the attachment points of the main shoulder muscles: subscapularis, supraspinatus, infraspinatus, teres minor and deltoid (Figure 1a). Second, a wrapping algorithm was applied to identify the points where muscles wrap around bones or potential implants. Finally, the accuracy of the automatically indicated landmarks and its effect on the

${ }^{*}$ Both authors contributed equally to this manuscript 
muscle elongation were evaluated by comparing the manually indicated landmarks with the landmarks identified through the SSM.

The accuracy evaluation was performed by a leave-one-out (LOO) cross-validation. For each selected humerus, a new 'sub'-SSM was created from the 39 remaining humeri, while excluding the selected bone. After identifying the muscle attachment points with the 'sub'-SSM, wrapping points were determined and the muscle lengths were computed. Next, a reverse humerus and glenoid implant were virtually implanted, muscle lengths were recomputed and muscle elongations relative to the preoperative situation were calculated (Figure 1b).
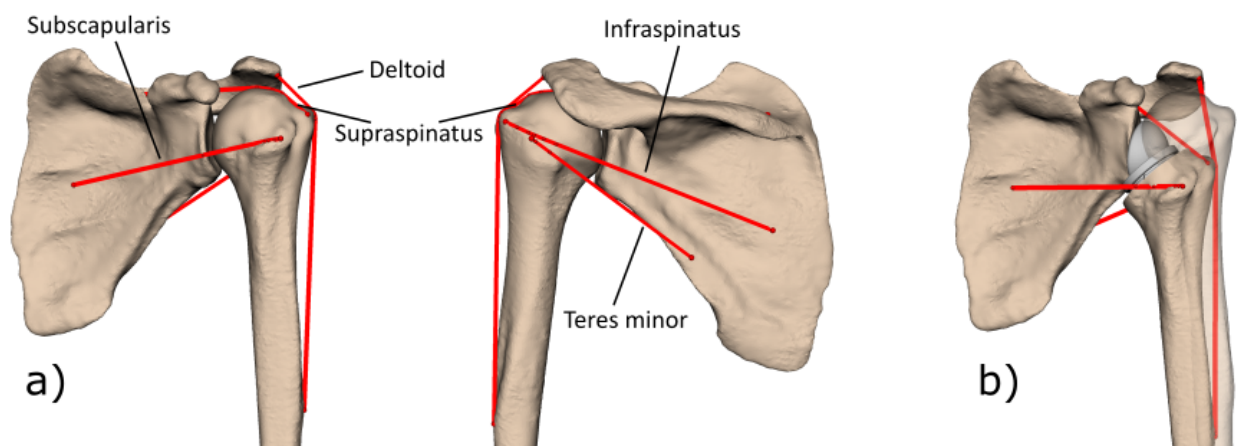

Figure 1: (a) Shoulder muscles. (b) Reverse shoulder implant: pre (transparent) vs post-op model.
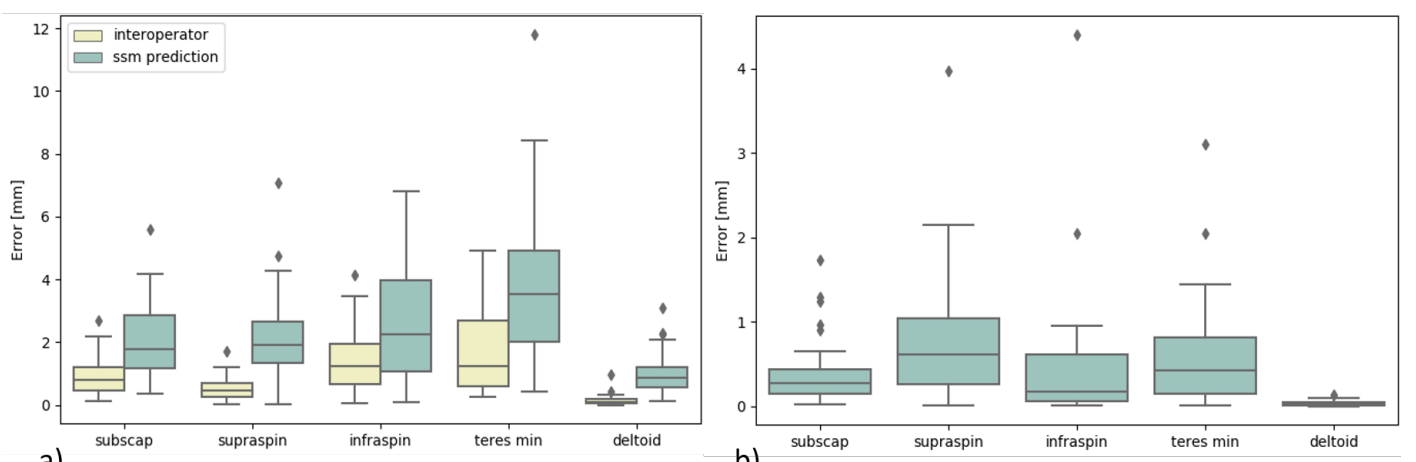

a)

b)

\section{Results}

The median interoperator error for the attachment points was below $2 \mathrm{~mm}$ for all muscles. The SSM prediction errors, although showing a similar trend, were consistently higher, with a maximum median error of almost $4 \mathrm{~mm}$ for the teres minor landmark (Figure 2a).

For all muscle elongations the median error was smaller than $1 \mathrm{~mm}$, while $95 \%$ of all the error measurements were below $2 \mathrm{~mm}$ (Figure 2b).

Figure 2: (a) Landmarks: interoperator variability and SSM prediction error. (b) Muscle elongation errors 


\section{Discussion}

A novel method to automatically estimate muscle elongation, based on a statistical shape modelling approach, was presented. For all the landmarks, the SSM prediction errors are higher than the interoperator variability. The higher inaccuracy is partly due to the mesh size of the SSM, which limits the prediction accuracy. However, when compared to previous studies (Kaptein \& van der Helm, 2004; Pellikaan et al., 2014), our method shows better estimations.

The errors on muscle elongation were substantially smaller than the errors on the landmarks. The low errors on elongation ( $75 \%$ of the data points below $1 \mathrm{~mm}$ ) suggest that the presented automated workflow is a promising tool for allowing surgeons to evaluate patient-specific muscle elongations during preoperative planning.

Although the evaluation was limited to healthy joints, this method allows to easily process large datasets and to potentially find a correlation between muscle elongations and postoperative outcome.

\section{References}

Kaptein, B. L., \& van der Helm, F. C. T. (2004). Estimating muscle attachment contours by transforming geometrical bone models. Journal of Biomechanics, 37(3), 263-273. https://doi.org/10.1016/j.jbiomech.2003.08.005

Lädermann, A., Edwards, T. B., \& Walch, G. (2014). Arm lengthening after reverse shoulder arthroplasty: A review. International Orthopaedics, 38(5), 991-1000. https://doi.org/10.1007/s00264013-2175-z

Marra, M. A., Vanheule, V., Fluit, R., Koopman, B. H. F. J. M., Rasmussen, J., Verdonschot, N., \& Andersen, M. S. (2015). A Subject-Specific Musculoskeletal Modeling Framework to Predict In Vivo Mechanics of Total Knee Arthroplasty. Journal of Biomechanical Engineering, 137(2), 020904. https://doi.org/10.1115/1.4029258

Pellikaan, P., van der Krogt, M. M., Carbone, V., Fluit, R., Vigneron, L. M., Van Deun, J., Verdonschot, N., \& Koopman, H. F. J. M. (2014). Evaluation of a morphing based method to estimate muscle attachment sites of the lower extremity. Journal of Biomechanics, 47(5), 1144-1150. https://doi.org/10.1016/j.jbiomech.2013.12.010

Plessers, K., Vanden Berghe, P., Van Dijck, C., Wirix-Speetjens, R., Debeer, P., Jonkers, I., \& Vander Sloten, J. (2018). Virtual reconstruction of glenoid bone defects using a statistical shape model. Journal of Shoulder and Elbow Surgery, 27(1), 160-166. https://doi.org/10.1016/j.jse.2017.07.026

\section{Acknowledgements}

This project has received funding from the European Union's Horizon 2020 research and Innovative programme under the Marie Skłodowska-Curie grant agreement No 722535. 